\section{Zebrafish Hairy/Enhancer of split protein links FGF signaling to cyclic gene expression in the periodic segmentation of somites}

\author{
Akinori Kawamura, ${ }^{1,6}$ Sumito Koshida, ${ }^{1,2,3,6}$ \\ Hiroko Hijikata, ${ }^{3}$ Takuya Sakaguchi, ${ }^{4}$ \\ Hisato Kondoh, ${ }^{3,5}$ and Shinji Takada ${ }^{1,2,3,7}$ \\ ${ }^{1}$ Okazaki Institute for Integrative Biosciences, National \\ Institutes of Natural Sciences, Okazaki, Aichi 444-8787, \\ Japan; ${ }^{2}$ Department of Molecular Biomechanics, The Graduate \\ University for Advanced Studies (SOKENDAI), Okazaki, \\ Aichi 444-8787, Japan; ${ }^{3}$ Kondoh Differentiation Signaling \\ Project, Exploratory Research for Advanced Technology \\ (ERATO)/SORST Program, Kondoh Research Team, Japan \\ Science and Technology Corporation (JST), Kinki Invention \\ Center, Sakyo-ku, Kyoto 606-8305, Japan; ${ }^{4}$ Department of \\ Biochemistry and Biophysics, Programs in Developmental \\ Biology, Genetics and Human Genetics, University of \\ California, San Francisco, San Francisco, California 94143, \\ USA; ${ }^{5}$ Graduate School of Frontier Biosciences, Osaka \\ University, Suita, Osaka 565-0871, Japan
}

Notch and fibroblast growth factor (FGF) signaling pathways have been implicated in the establishment of proper periodicity of vertebrate somites. Here, we show evidence that a Hes6-related hairy/Enhancer of split-related gene, her13.2, links FGF signaling to the Notchregulated oscillation machinery in zebrafish. Expression of her13.2 is induced by FGF-soaked beads and decreased by an FGF signaling inhibitor. her13.2 is required for periodic repression of the Notch-regulated genes her1 and her 7 , and for proper somite segmentation. Furthermore, Her13.2 augments autorepression of her1 in association with Her1 protein. Therefore, FGF signaling appears to maintain the oscillation machinery by supplying a binding partner, Her13.2, for Her1.

Supplemental material is available at http://www.genesdev.org.

Received December 17, 2004; revised version accepted March 31, 2005.

Somites are the morphologically distinct segmental units that are transiently formed during early vertebrate development and subsequently give rise to metameric and fundamental structures such as the vertebrae of the axial skeleton, their associated muscles, and tendons. The somites are subdivided from the anterior end of the unsegmented paraxial mesoderm, called the presomitic

[Keywords: Somite segmentation; FGF signaling; cyclic gene expression; hairy/Espl; negative feedback loop; zebrafish]

${ }^{6}$ These authors contributed equally to this work.

${ }^{7}$ Corresponding author.

E-MAIL stakada@nibb.ac.jp; FAX 81-564-59-5240.

Article and publication are at http://www.genesdev.org/cgi/doi/10.1101/ gad.1291205. mesoderm (PSM), and sequentially generated in an anterior to posterior direction in a rhythmic fashion at regular spatiotemporal intervals. The molecular mechanism underlying the periodical formation of somites is coupled to an internal oscillator, referred to as the "segmentation clock", which has been evidenced by the cyclic expression of genes in the PSM (Palmeirim et al. 1997; Maroto and Pourquie 2001). Most genes that exhibit a cyclic expression pattern in the PSM are involved in the Notch signaling pathway (Bessho and Kageyama 2003). Various hairy/Enhancer of split (Espl)-related basic helix-loop-helix (bHLH) genes (hairy-1, hairy-2, and Hey2 in the chicken; Hes1, Hes5, Hes7, and Hey2 in the mouse; her1 and her7 in the zebrafish) that are transcriptional targets of the Notch signaling are expressed in a dynamic pattern of stripes across the PSM in a posterior to anterior direction. In addition, Lunatic fringe, encoding a glycosyltransferase that modulates the Notch signaling in the chicken and mouse, and the Notch ligand deltaC in the zebrafish also show an oscillatory expression pattern in the PSM. These cyclic genes, as well as other components of the Notch signaling pathway, were shown to be required for the proper somite segmentation in mice (Hrabe de Angelis et al. 1997; Kusumi et al. 1998; Bessho et al. 2001) and zebrafish (Takke and CamposOrtega 1999; Holley et al. 2000, 2002; Henry et al. 2002; Oates and Ho 2002) by mutant analysis or morpholino oligo (MO)-mediated gene-knockdown experiments. Furthermore, the cyclic expression of Hes7, her1, and her7 requires their own activity, suggesting that a negative feedback loop involving these genes is a critical component of the oscillation machinery (Oates and Ho 2002; Bessho et al. 2003).

The oscillating and anteriorly propagating wave of gene expression, which is maintained in the posterior PSM, becomes fixed to cause segmentation in the anterior PSM. Activity gradients of signaling molecules, including fibroblast growth factor (FGF), Wnt, and retinoic acid are proposed to regulate the differentiation of PSM cells along the anteroposterior axis from a state permitting the oscillating gene expression to a state driving the segmentation program (Dubrulle et al. 2001; Sawada et al. 2001; Aulehla et al. 2003; Moreno and Kintner 2004). For instance, FGF signaling is the highest at the posterior end of the PSM with a gradual decrease toward the anterior, suggesting a role for FGF signaling in maintaining the characteristics of the posterior PSM cells (Dubrulle et al. 2001; Sawada et al. 2001). In fact, overexpression of FGF8 in the entire PSM of chick embryos causes an increase in the expression of Brachyury in the posterior PSM and suppresses morphological segmentation (Dubrulle et al. 2001). Furthermore, transient activation or inhibition of FGF signaling results in the formation of smaller or larger somites, respectively. Thus, FGF signaling appears to maintain the posterior characteristics, which allows the cyclic gene expression, and the level of FGF activity regulates the transition of the posterior PSM cells into segmental tissues and determines the position of the segment boundaries. However, the molecular mechanism by which FGF signaling permits the cyclic gene expression in the posterior PSM has been totally unknown.

Here we focused on a zebrafish hairy/Espl gene, 
her13.2, which is expressed in the posterior PSM. Our results indicate that FGF signaling allows the cyclic gene expression by inducing her13.2 in the posterior PSM.

\section{Results and Discussion}

To gain a better insight into the molecular mechanism underlying the segmentation process, we identified genes specifically expressed in the PSM by an in situ hybridization screening of mRNA expressed in the PSM to tailbud region of zebrafish embryos (18-21-somite stages). Among these genes, her13.2, coding for a bHLH protein closely related to mammalian Hes6 (Bae et al. 2000; Koyano-Nakagawa et al. 2000; Sieger et al. 2004), exhibited posterior-to-anterior decrease in expression, analogous to the FGF signaling gradient, in the posterior PSM and tailbud. Expression of her13.2 was first observed in the blastoderm margin at the shield stage (Fig. 1A), and then became restricted to the posterior region at the tailbud stage, being excluded from the axial mesoderm (Fig. 1B). As segmentation proceeded, the transcripts became specifically localized to the posterior PSM and tailbud (Fig. 1C-F). Expression of her13.2 was strong at the tailbud and gradually decreased along the PSM (Fig. 1D). In contrast to other her genes, e.g., her1 and her7, which are known to exhibit oscillatory expression in the PSM and tailbud (Holley et al. 2000; Sawada et al. 2000; Henry et al. 2002; Oates and Ho 2002; Gajewski et al. 2003), her13.2 is unique for its nonoscillatory expression.

Since the graded axial distribution of her13.2 mRNA in the posterior PSM resembled that of FGF activity, we examined whether FGF signaling was involved in the expression of her13.2. With a brief treatment of embryos with SU5402, an FGF receptor kinase inhibitor (Mohammadi et al. 1997), expression of her13.2 in the PSM was drastically reduced, whereas DMSO-treated control em-

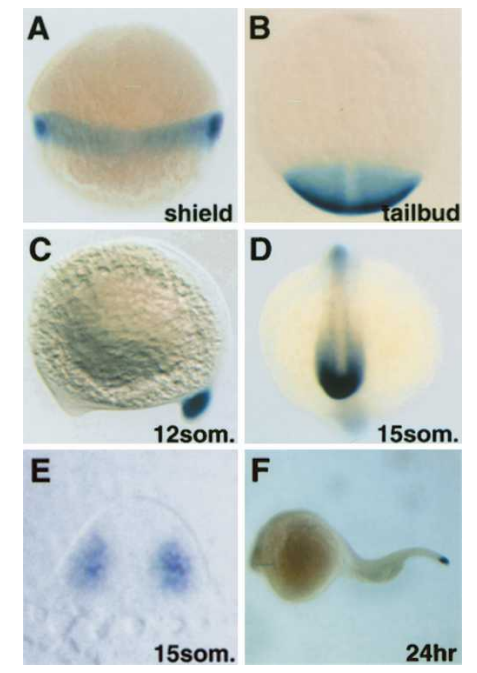

Figure 1. Zebrafish her13.2 mRNA is expressed in the posterior PSM and tailbud. $(A, C, F)$ Lateral view. $(B, D)$ Dorsal view. Wholemount in situ hybridization was carried out by using her13.2 antisense probe and embryos at the developmental stages shown at the bottom right of each panel. her13.2 expression is first seen at the blastoderm margin at the shield stage. During the segmentation period, the signal remains confined to the posterior PSM and tailbud. (E) Transverse section of a stained embryo at the level of the posterior PSM. A signal is observed in the paraxial mesoderm.

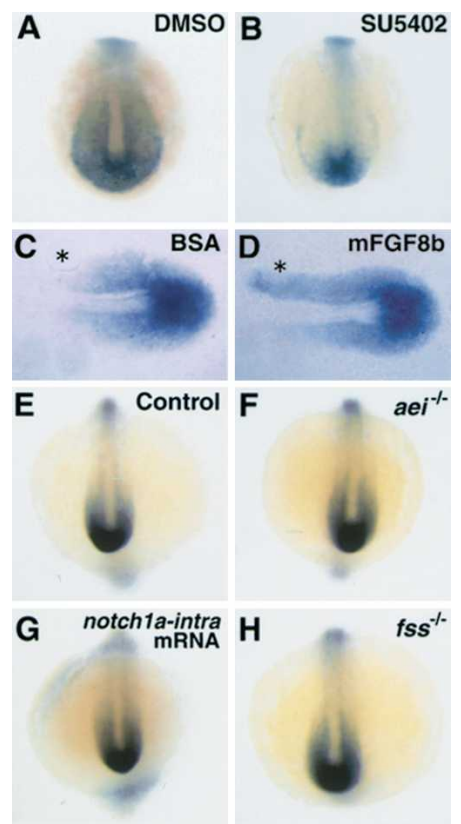

Figure 2. Expression of her13.2 is regulated by FGF signaling. Each panel is shown from the dorsal aspect. $(A, B)$ Transient inhibition of FGF signaling reduces the expression of her13.2 in the posterior PSM. her13.2 expression in embryos treated with DMSO $(n=6 ; 0 \%$ affected) and SU5402 at the concentration of $660 \mathrm{\mu M}(n=6 ; 100 \%$ affected). Reduced expression of her13.2 was observed in embryos treated with SU5402 at any concentration that caused expansion of

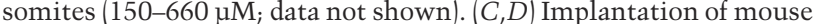
recombinant FGF8b-soaked bead leads to the ectopic expression of her13.2. her13.2 expression in flat-mounted embryos with BSAsoaked $(n=6 ; 0 \%$ affected) or mFGF8b-soaked $(n=9 ; 100 \%$ affected $)$ bead. To minimize possible artifacts, beads were implanted lateral to the paraxial mesoderm. The anterior is left, and asterisks show the position of implanted beads. $(E-H)$ Independence of her13.2 expression from Notch signaling and $f_{S s} / t b \times 24$. Expression pattern of her13.2 was comparable between sibling $(n=16 ; 0 \%$ affected $)$ and aei/deltaD $D^{-/-}(n=11 ; 0 \%$ affected) embryos. her13.2 expression was not altered in embryos injected with mRNA encoding a constitutively active form of notch1a $(n=20 ; 0 \%$ affected), although ectopic expression of her1 was observed in the entire PSM of these embryos ( $n=23 ; 87 \%$ affected). her13.2 expression was also not altered in fss $/$ tb $\times 24^{-/-}$mutants $(n=7 ; 0 \%$ affected).

bryos maintained the normal expression of her13.2 (Fig. 2A,B). Conversely, implantation of an mFGF8bsoaked bead into the anterior PSM caused anterior expansion of the expression domain of her13.2, whereas a bovine serum albumin (BSA)-soaked control bead did not affect her13.2 expression (Fig. 2C,D). On the other hand, Notch signaling, which is known to activate hairy/Esplrelated genes showing oscillatory expression in the PSM (Bessho and Kageyama 2003), did not affect the expression of her13.2 in the PSM. Expression of her13.2 in aei/ deltaD ${ }^{-/-}$embryos, as well as in embryos injected with mRNA encoding the intracellular domain of notch1a, which can activate the Notch signaling constitutively (Takke and Campos-Ortega 1999), appeared indistinguishable from that in normal embryos (Fig. 2E-G). Furthermore, expression of her13.2 was not significantly altered in fss/tbx24 mutant embryos (Fig. $2 \mathrm{H}$ ). These results indicate that her13.2 functions downstream of FGF signaling in the PSM, but is not under direct regulation by Notch signaling, or Tbx24 transcription factor, in contrast to other hairy/Espl-related genes. 


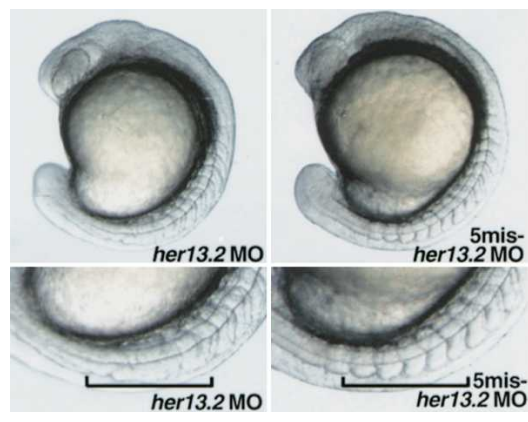

Figure 3. Altered somite patterning in her13.2 MO-injected embryos. Lateral views of her13.2 MO-injected and 5mis-her13.2 MOinjected embryos at the 12-somite stage. In her13.2 MO-injected embryos, the boundaries of somites are disrupted after the proper segmentation of the first eight somites, whereas no apparent defect is seen in 5mis-her13.2 MO-injected embryos. Injected embryos were incubated at $23.5^{\circ} \mathrm{C}$ after the dome stage $(4.5 \mathrm{hpf})$, because this segmental defect was more prominent after this stage.

To investigate the involvement of her13.2 in somite development, we performed antisense MO-mediated gene-knockdown experiments. Injection of two distinct antisense MOs specific for her13.2 resulted in disruption of the regular segmentation of somites posterior to the first seven to nine somites (Fig. 3). This phenotype closely resembled the phenotypes of previously identified segmentation mutants, such as after eight, deadly

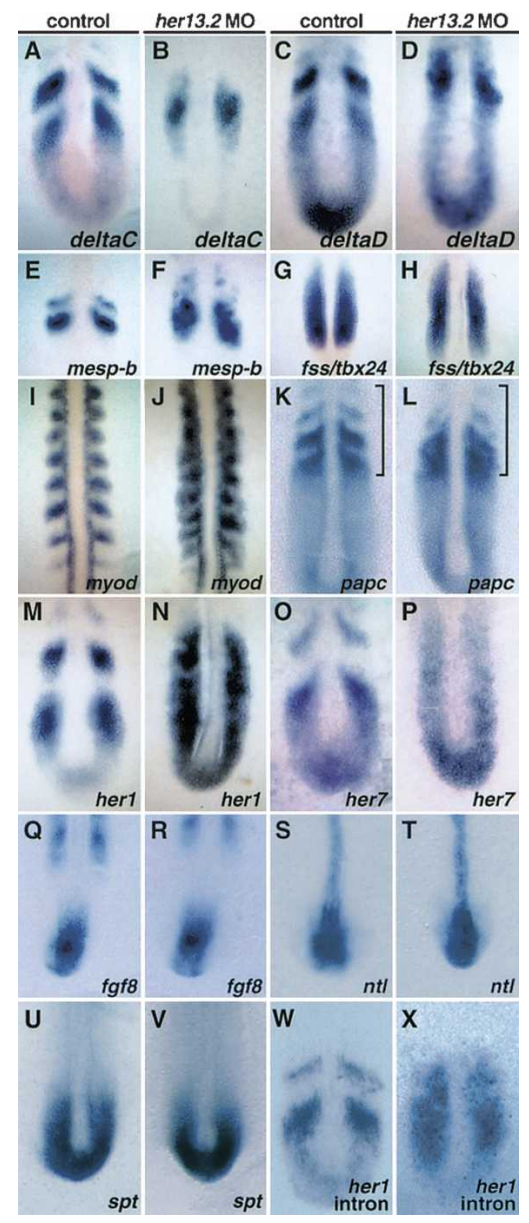

seven, and mind bomb (van Eeden et al. 1996). In contrast, MOs carrying five base substitutions in the her13.2-specific MOs caused no such defect. Coinjection of variant her13.2 mRNA lacking the her13.2 MO-target sequence rescued embryos from this segmental defect in a dose-dependent manner (Supplementary Table 1). Thus, her13.2 is required for the proper segmentation of somites in the posterior trunk. The segmental defect caused by her13.2-specific MOs was confirmed by analysis of the expression of a series of genes characteristically involved in the segmentation process. For instance, del$t a C$, deltaD, and mesp- $b$, which are normally expressed in a segmental pattern, were expressed in a scattered pattern in the anterior PSM of her13.2 MO-injected embryos (Fig. 4A-F), as typically observed in embryos defective in the segmentation process (Jiang et al. 2000; Sawada et al. 2001; Holley et al. 2002). In contrast, the expression of $f s s / t b \times 24$, which is normally expressed uniformly in the anterior and intermediate PSM (Nikaido et al. 2002), was not affected (Fig. 4G,H). Rostrocaudal polarity of somites was also perturbed in her13.2 MO-injected embryos. Expression of myod, which is normally confined to the posterior region of somites (Weinberg et al. 1996), was expanded (Fig. 4I,J), whereas that of paraxial protocadherin (papc), which is normally expressed in the PSM and anterior region of somites (Yamamoto et al. 1998), was scattered and reduced (Fig. 4K,L). These results indicate that her13.2 function is essential for the process of somite segmentation.

In the process of segmentation, hairy/Espl-related genes her1 and her 7 in the zebrafish and Hes7 in the mouse play essential roles in the segmentation machinery, because disruption of these genes results in defects in the segmentation and the oscillatory expression of the cyclic genes (Bessho et al. 2001; Henry et al. 2002; Holley

Figure 4. Ectopic expression of her1 and her 7 in the PSM of her13.2 MO-injected embryos. Expression analysis of her13.2 MO-injected embryos by using probes indicated at the bottom right of each panel. Embryos were fixed at the 10-12-somite stages. Stained embryos were flat-mounted. $(A-F)$ The segmental expression of deltaC ( $n=14 ; 86 \%$ affected), deltaD ( $n=14 ; 93 \%$ affected), and mesp- $b$ $(n=14 ; 86 \%$ affected) has vanished. Instead, scattered expression is observed in the anterior PSM of her13.2 MO-injected embryos. $(G, H)$ $f_{S s} / t b \times 24$, normally expressed in the anterior and intermediate PSM, is not altered $(n=15 ; 0 \%$ affected). (I-L) The polarity of somites is posteriorized in her13.2 morphants. The distribution of myod, expressed in the posterior region of somites, is expanded $(n=14 ; 93 \%$ affected). That of papc, expressed in the PSM and anterior part of somites, is scattered $(n=15 ; 87 \%$ affected) and emphasized by the bracket. $(M-P)$ Cyclic expression patterns of her1 and her7, categorized into three phases (Jiang et al. 2000; Oates and Ho 2002), are observed in the control embryos $(n=16$; phase I, 5; phase II, 5; phase III, 6; and $n=10$; phase I, 3; phase II, 3; phase III, 4, respectively). Expression pattern of her 1 in $M$ represents phase I, and that of her 7 in $O$ represents phase II. In her13.2 morphants, however, expression of her1 ( $n=23 ; 87 \%$ affected) and her7 ( $n=13 ; 85 \%$ affected) is seen in the entire PSM, distinct from the three phases observed in the control embryos. $(Q-V)$ fgf8 $(n=13 ; 0 \%$ affected $)$ and FGF-downstream genes such as $n t l(n=11 ; 0 \%$ affected $)$ and $\operatorname{spt}(n=13 ; 0 \%$ affected) are normally expressed in the posterior region of her13.2 MO-injected embryos. $(W, X)$ Transcriptionally active state of her1 in the PSM of her13.2 MO-injected embryos. Cyclic expression of her1 primary transcripts is detected in control embryos by use of a her 1 intron probe $(n=21$; phase I, 6; phase II, 7; phase III, 8$)$. Expression pattern of nascent her1 in $W$ represents phase II. However, in her13.2 MO-injected embryos, her1 nascent mRNA is observed in the entire PSM ( $n=21 ; 81 \%$ affected), as found with the her1 exon probe (Fig. 4N). 
et al. 2002; Oates and Ho 2002; Gajewski et al. 2003). These genes also exhibit periodical expression in the posterior PSM, the periodicity of which is generated by a negative feedback loop composed of repression of the genes by their own encoded proteins and break of this repression by ubiquitination-mediated degradation of the proteins (Oates and Ho 2002; Bessho et al. 2003; Hirata et al. 2004). Interestingly, in her13.2 MO-injected embryos, the expression of her1 and her 7 was observed in the entire region of the PSM (Fig. 4M-P), as observed in embryos deficient for her1 and/or her7 (Henry et al. 2002; Holley et al. 2002; Oates and Ho 2002; Gajewski et al. 2003), although the expression level was variable among cells in the PSM of the her13.2-deficient embryos. In contrast, the expression of $f g f 8$, no tail (ntl), and spade tail (spt), which exhibit nonoscillating expression in the posterior PSM, was not significantly affected (Fig. 4Q-V). Thus, her13.2 is required for the cyclic repression of her1 and her7 expression. On the other hand, expression of another cyclic gene, deltaC, was decreased by injecting her13.2-specific MO (Fig. 4A,B), indicating that her13.2 acts differently on the expression of this gene. To determine whether the up-regulated expression of her1 and her7 genes was caused by decreased repression of their transcription or by stabilization of their mRNAs, we examined the distribution of her1 nascent transcripts by using a her1 intron probe. In normal embryos, the segmental expression of nascent her1 transcripts was observed (Fig. 4W), as previously reported (Gajewski et al. 2003). On the other hand, in her13.2 MO-injected embryos, the signal was widespread in the posterior PSM, as was the case when a her1 exon probe was used (Fig. 4X). Thus, her13.2 plays an essential role in cyclic repression of her1 transcription in the posterior PSM.

We next addressed how Her13.2 is involved in repression of the her1 gene transcription by conducting an in vitro luciferase reporter assay. The expression of the luciferase gene under the control of an $8.6-\mathrm{kb}$ her1 promoter is sufficient for the normal expression of the her1 gene in the PSM (Gajewski et al. 2003). This promoter activity was repressed by Herl, but not affected by Her13.2, in 293T cells (Fig. 5A). Under this experimental condition, Her13.2 enhanced the self-repression activity of Her1 (Fig. 5B). Thus, Her13.2 can act as a transcriptional repressor with Her1, and enhancement of her1 repression by Her13.2 may be required for the periodical repression of her1 in the posterior PSM. To function as a transcriptional repressor, Her13.2 appears to require another bHLH protein as a binding partner, because Her13.2 has a uniquely short loop region within its HLH domain, causing an inability to bind to the target sequences (Bae et al. 2000). Thus, it is possible that Her13.2 may enhance the self-repression of her1 through formation of Her1-Her13.2 heterodimer complex. In fact, an in vitro pulldown assay indicated that Her13.2 protein interacted physically with the Herl protein (Fig. 5C). These results demonstrate that Her13.2 can strongly repress her1 expression, presumably through complex formation with Her1.

Taken together, our findings indicate that FGF signaling induces the expression of her13.2 in the posterior PSM, and, in turn, Her13.2 protein participates in the periodic repression of the Notch-regulated genes, her1 and her7, presumably through formation of a transcriptional repressor complex with at least one of these Her proteins, Her1. FGF signaling has been shown to main-

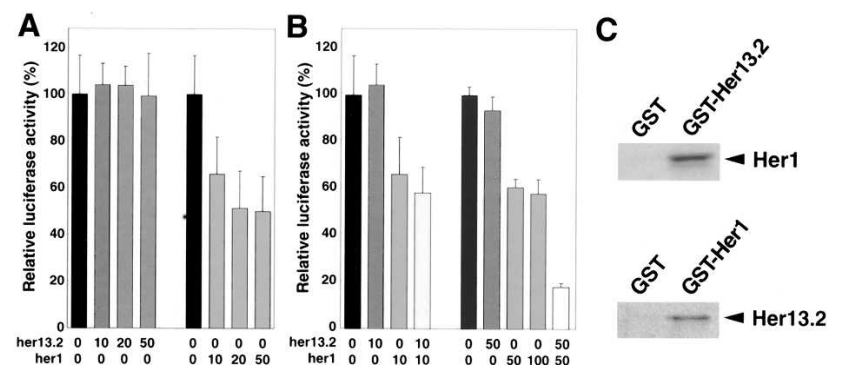

Figure 5. Her13.2 interacts with Her1 and synergistically represses the transcriptional activity of the her1 promoter. $(A, B)$ Her13.2 alone has little transcriptional effect on the her1 promoter, but synergistically represses her 1 activity in the presence of Her1. Luciferase assay using $293 \mathrm{~T}$ cells was conducted to examine the effects of introduction of her13.2 and her1 on the $8.6-\mathrm{kb}$ fragment upstream of the translational start site of her 1 fused to the firefly luciferase gene. The amounts of $\mathrm{pCS}^{+}$her13.2 and pCS2 $2^{+}$her1 plasmids (in nanograms) used for transfection are shown under each bar. Total amount of DNA was adjusted with $\mathrm{pCS}^{+}$vector to be equal. All experiments were normalized for transfection efficiency by cotransfection with an expression vector for Renilla luciferase. The average normalized firefly luciferase without $\mathrm{pCS} 2^{+}$her 13.2 and $\mathrm{pCS} 2^{+}$her 1 was set at $100 \%$, and the error bar represents the standardd. deviation. All transfections were performed in triplicate, and similar results were obtained in at least duplicate experiments. $(C)$ Interaction between Her13.2 and Herl in vitro. A pulldown assay was performed with in vitro labeled Her1 or Her13.2 proteins and purified Her13.2-GST or Her1-GST fusion proteins on glutathione Sepharose 4B, respectively. GST-Her13.2, not GST alone, associates with Her1 specifically, and vice versa. These specific interactions were confirmed in duplicate experiments.

tain posterior PSM cells in a state that allows the oscillatory expression of the cyclic genes, and also to be associated with expression of nonperiodic genes, e.g., $f g f 8$, $n t l$, and spt (Dubrulle et al. 2001; Sawada et al. 2001), whose expression was not obviously altered in her13.2 MO-injected embryos. Therefore, it is likely that FGF signaling is transmitted through several different molecular pathways in the posterior PSM, and that Her13.2 mediates specifically one of the roles of FGF signaling, the regulation of the cyclic genes. On the other hand, we cannot conclude at present whether her13.2 expression is sufficient for this role of FGF signaling. Overexpression of her13.2 did not give us an answer to this question, because the overexpression resulted in malformation of the presomitic mesoderm, which inhibited the precise analysis of the periodic gene expression (data not shown).

In addition to FGF signaling, Wnt signaling has been indicated to regulate the oscillation machinery (Aulehla et al. 2003). Recently, two groups reported that Wnt signaling directly regulates the expression of Dll1, a component of oscillation machinery in mouse embryos (Galceran et al. 2004; Hofmann et al. 2004). Thus, two molecular systems, which are individually activated by FGF or Wnt signaling, regulate the oscillation machinery. This may lead to speculation that these two regulatory systems coordinate in the maintenance of the oscillation machinery. However, it is uncertain whether these two regulatory systems function simultaneously in a particular species. In mouse, in contrast to zebrafish, the only structural counterpart of her13.2, Hes6, is not expressed in the PSM (Bae et al. 2000). Furthermore, in the zebrafish, despite a number of findings in the mouse, the involvement of Wnt signaling in the segmentation clock 
remains obscure. Thus, we can also speculate that different species may establish their own ways to transmit the signaling gradient to the segmentation clock. We expect that this issue will be solved by more extensive analysis in the future.

\section{Materials and methods}

Fish

All the experiments except mutant analysis were performed using the TL2 inbred line (Kishimoto et al. 2004). The mutant alleles used in this study are after eight $\left(a e^{\text {tr2333 }}\right)$ and fused somites $\left(f_{s s^{t i l}}\right)$.

Construction of cDNA library, screening, and isolation of her13.2 A cDNA library was constructed with mRNA prepared from the PSM to tailbud region of zebrafish embryos at 18-21-somite stages. For collection of cDNA clones expressed in the PSM and tailbud region, a high-throughput in situ hybridization screening was performed in 96-well plates. The precise procedure for this screening will be described elsewhere. One of the cDNA clones obtained by this screening was a partial cDNA fragment of her13.2. Using this fragment as a probe, we obtained an $\sim 1.2-\mathrm{kb}$ cDNA from a 15-19 hours post-fertilization (hpf) cDNA library.

Whole-mount in situ hybridization

Whole-mount in situ hybridization was performed as described (Nikaido et al. 1997). For the her1 intron probe, the 1.4-kb genomic region corresponding to the her1 intron was obtained by PCR with the following

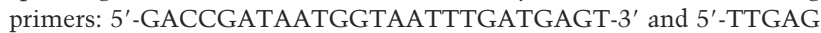
ATGAAATGAAAAGGAAAAG-3', and hybridization was carried out as described (Gajewski et al. 2003).

Antisense MO and mRNA synthesis of her13.2

The sequences of MO used in this study are as follows: her13.2 MO1, 5'-TGCAGTTCAGGACGCTTGAATGGG-3'; 5mis-her13.2 MOl, 5'TGCAcTTgAGGACcCTTGtATcGG-3'; her13.2 MO2, 5'-GGCAGATG GTCGGCGGTTCAGTTC-3'; 5mis-her13.2 MO2, 5'-GGgAGtTGGTC GaCGGTaCAcTTC- $3^{\prime}$. The DNA fragment encoding the entire ORF of her13.2 was inserted into pCS2+ vector. Capped her13.2 mRNA was produced with an mMessage mMachine (Ambion).

SU5402 treatment and bead implantation

These experiments were performed essentially as described (Sawada et al. 2001).

\section{Luciferase reporter assay}

Reporter gene pGL3-herl was constructed by inserting an 8.6-kb fragment upstream of the translational initiation site of zebrafish her1 into the upstream of firefly luciferase gene in pGL3 Basic vector (Promega). About $1.0 \times 10^{4} 293 \mathrm{~T}$ cells were seeded into each well of a 24-well collagen-coated plate (Iwaki). pCS2 ${ }^{+}, \mathrm{pCS} 2^{+}$her1, and $\mathrm{pCS} 2^{+}$her13.2 vector with pGL3-her1 (100 ng) and pRL-TK $(0.2 \mathrm{ng})$ were transiently introduced into the cells using fugene6 (Roche). After incubation for $24 \mathrm{~h}$, the cells were lysed, and the lysates were assayed with a Dual-Luciferase reporter assay system (Promega).

\section{GST pulldown assay}

To generate GST-Her13.2 and GST-Her1 proteins, the DNA fragment corresponding to the entire amino acid sequence of either her13.2 or her1 was inserted in-frame into pGEX4T-3 vector (Amersham). GST fusion proteins were purified on glutathione Sepharose 4B (Amersham). For synthesis of in vitro labeled proteins, $1 \mu \mathrm{g}$ of either pCS2 ${ }^{+}$her13.2 or pCS2 + herl containing the complete amino acid sequence of her1 was added to a TNT Quick coupled Transcription/Translation Systems (Promega) in the presence of $\left[{ }^{35} \mathrm{~S}\right]$-labeled methionine (Amersham). In $1 \mathrm{~mL}$ of $2 \% \mathrm{BSA}$ in $\mathrm{PBS}(-), 5 \mu \mathrm{L}$ of in vitro translation products was mixed and incubated with $5 \mu \mathrm{g}$ of either GST, GST-Her13.2, or GST-Herl for $60 \mathrm{~min}$ at $4^{\circ} \mathrm{C}$. Following extensive washing, the bound proteins were separated in a $12 \%$ SDS-polyacrylamide gel.

\section{Acknowledgments}

We thank B. Appel for the 15-19 hpf cDNA library; J. Campos-Ortega for pCS2 $2^{+}$her1 and pCS2 ${ }^{+}$notchla-intra plasmids; D. Tautz for her1 pro- moter; Y.J. Jiang for aei mutant and probe; M. Furutani-Seiki for $f_{s s}$ mutant; H. Takeda, R. Kageyama, and M. Nikaido for probes; Y. Bessho for in situ hybridization protocol using intron probe; and A. Sawada for technical advice. We are grateful to Y. Kishimoto for discussions. This work was supported by a grant-in-aid for scientific research from the Ministry of Education, Science, Culture, and Sports of Japan to S.T. and S.K., and by grants from the Japan Science and Technology Corporation and Mitsubishi Foundation to S.T. A.K. is a research fellow of the Japan Society for the Promotion of Science.

\section{References}

Aulehla, A., Wehrle, C., Brand-Saberi, B., Kemler, R., Gossler, A. Kanzler, B., and Herrmann, B.G. 2003. Wnt3a plays a major role in the segmentation clock controlling somitogenesis. Dev. Cell 4: 395-406.

Bae, S., Bessho, Y., Hojo, M., and Kageyama, R. 2000. The bHLH gene Hes6, an inhibitor of Hes1, promotes neuronal differentiation. Development 127: 2933-2943.

Bessho, Y. and Kageyama, R. 2003. Oscillations, clocks and segmentation. Curr. Opin. Genet. Dev. 13: 379-384.

Bessho, Y., Sakata, R., Komatsu, S., Shiota, K., Yamada, S., and Kageyama, R. 2001. Dynamic expression and essential functions of Hes7 in somite segmentation. Genes \& Dev. 15: 2642-2647.

Bessho, Y., Hirata, H., Masamizu, Y., and Kageyama, R. 2003. Periodic repression by the bHLH factor Hes7 is an essential mechanism for the somite segmentation clock. Genes \& Dev. 17: 1451-1456.

Dubrulle, J., McGrew, M.J., and Pourquie, O. 2001. FGF signaling controls somite boundary position and regulates segmentation clock control of spatiotemporal Hox gene activation. Cell 106: 219-232.

Gajewski, M., Sieger, D., Alt, B., Leve, C., Hans, S., Wolff, C., Rohr, K.B., and Tautz, D. 2003. Anterior and posterior waves of cyclic herl gene expression are differentially regulated in the presomitic mesoderm of zebrafish. Development 130: 4269-4278.

Galceran, J., Sustmann, C., Hsu, S.C., Folberth, S., and Grosschedl, R. 2004. LEF1-mediated regulation of $\delta$-like1 links Wnt and Notch signaling in somitogenesis. Genes \& Dev. 18: 2718-2723.

Henry, C.A., Urban, M.K., Dill, K.K., Merlie, J.P., Page, M.F., Kimmel, C.B., and Amacher, S.L. 2002. Two linked hairy/Enhancer of splitrelated zebrafish genes, herl and her7, function together to refine alternating somite boundaries. Development 129: 3693-3704.

Hirata, H., Bessho, Y., Kokubu, H., Masamizu, Y., Yamada, S., Lewis, J., and Kageyama, R. 2004. Instability of Hes7 protein is crucial for the somite segmentation clock. Nat. Genet. 36: 750-754.

Hofmann, M., Schuster-Gossler, K., Watabe-Rudolph, M., Aulehla, A., Herrmann, B.G., and Gossler, A. 2004. WNT signaling, in synergy with T/TBX6, controls Notch signaling by regulating Dll1 expression in the presomitic mesoderm of mouse embryos. Genes \& Dev. 18: 2712-2717.

Holley, S.A., Geisler, R., and Nusslein-Volhard, C. 2000. Control of her1 expression during zebrafish somitogenesis by a $\delta$-dependent oscillator and an independent wave-front activity. Genes \& Dev. 14: 16781690.

Holley, S.A., Julich, D., Rauch, G.J., Geisler, R., and Nusslein-Volhard, C. 2002. herl and the notch pathway function within the oscillator mechanism that regulates zebrafish somitogenesis. Development 129: $1175-1183$.

Hrabe de Angelis, M., McIntyre II, J., and Gossler, A. 1997. Maintenance of somite borders in mice requires the $\delta$ homologue DIIl. Nature 386: 717-721.

Jiang, Y.J., Aerne, B.L., Smithers, L., Haddon, C., Ish-Horowicz, D., and Lewis, J. 2000. Notch signalling and the synchronization of the somite segmentation clock. Nature 408: 475-479.

Kishimoto, Y., Koshida, S., Furutani-Seiki, M., and Kondoh, H. 2004. Zebrafish maternal-effect mutations causing cytokinesis defect without affecting mitosis or equatorial vasa deposition. Mech. Dev. 121: 79-89.

Koyano-Nakagawa, N., Kim, J., Anderson, D., and Kintner, C. 2000. Hes6 acts in a positive feedback loop with the neurogenins to promote neuronal differentiation. Development 127: 4203-4216.

Kusumi, K., Sun, E.S., Kerrebrock, A.W., Bronson, R.T., Chi, D.C., Bulotsky, M.S., Spencer, J.B., Birren, B.W., Frankel, W.N., and Lander, E.S. 1998. The mouse pudgy mutation disrupts $\delta$ homologue Dll3 and initiation of early somite boundaries. Nat. Genet. 19: 274-278. 
Maroto, M. and Pourquie, O. 2001. A molecular clock involved in somite segmentation. Curr. Top. Dev. Biol. 51: 221-248.

Mohammadi, M., McMahon, G., Sun, L., Tang, C., Hirth, P., Yeh, B.K., Hubbard, S.R., and Schlessinger, J. 1997. Structures of the tyrosine kinase domain of fibroblast growth factor receptor in complex with inhibitors. Science 276: 955-960.

Moreno, T.A. and Kintner, C. 2004. Regulation of segmental patterning by retinoic acid signaling during Xenopus somitogenesis. Dev. Cell 6: 205-218.

Nikaido, M., Tada, M., Saji, T., and Ueno, N. 1997. Conservation of BMP signaling in zebrafish mesoderm patterning. Mech. Dev. 61: 75-88.

Nikaido, M., Kawakami, A., Sawada, A., Furutani-Seiki, M., Takeda, H., and Araki, K. 2002. Tbx24, encoding a T-box protein, is mutated in the zebrafish somite-segmentation mutant fused somites. Nat. Genet. 31: 195-199.

Oates, A.C. and Ho, R.K. 2002. Hairy/E(spl)-related (Her) genes are central components of the segmentation oscillator and display redundancy with the $\delta /$ Notch signaling pathway in the formation of anterior segmental boundaries in the zebrafish. Development 129: 2929-2946.

Palmeirim, I., Henrique, D., Ish-Horowicz, D., and Pourquie, O. 1997. Avian hairy gene expression identifies a molecular clock linked to vertebrate segmentation and somitogenesis. Cell 91: 639-648.

Sawada, A., Fritz, A., Jiang, Y., Yamamoto, A., Yamasu, K., Kuroiwa, A., Saga, Y., and Takeda, H. 2000. Zebrafish Mesp family genes, mesp-a and mesp-b are segmentally expressed in the presomitic mesoderm, and Mesp-b confers the anterior identity to the developing somites. Development 127: 1691-1702.

Sawada, A., Shinya, M., Jiang, Y.J., Kawakami, A., Kuroiwa, A., and Takeda, H. 2001. Fgf/MAPK signalling is a crucial positional cue in somite boundary formation. Development 128: 4873-4880.

Sieger, D., Tautz, D., and Gajewski, M. 2004. her11 is involved in the somitogenesis clock in zebrafish. Dev. Genes Evol. 214: 393-406.

Takke, C. and Campos-Ortega, J.A. 1999. her1, a zebrafish pair-rule like gene, acts downstream of notch signalling to control somite development. Development 126: 3005-3014.

van Eeden, F.J., Granato, M., Schach, U., Brand, M., Furutani-Seiki, M., Haffter, P., Hammerschmidt, M., Heisenberg, C.P., Jiang, Y.J., Kane, D.A., et al. 1996. Mutations affecting somite formation and patterning in the zebrafish, Danio rerio. Development 123: 153-164.

Weinberg, E.S., Allende, M.L., Kelly, C.S., Abdelhamid, A., Murakami, T., Andermann, P., Doerre, O.G., Grunwald, D.J., and Riggleman, B. 1996. Developmental regulation of zebrafish MyoD in wild-type, no tail and spadetail embryos. Development 122: 271-280.

Yamamoto, A., Amacher, S.L., Kim, S.H., Geissert, D., Kimmel, C.B., and De Robertis, E.M. 1998. Zebrafish paraxial protocadherin is a downstream target of spadetail involved in morphogenesis of gastrula mesoderm. Development 125: 3389-3397. 


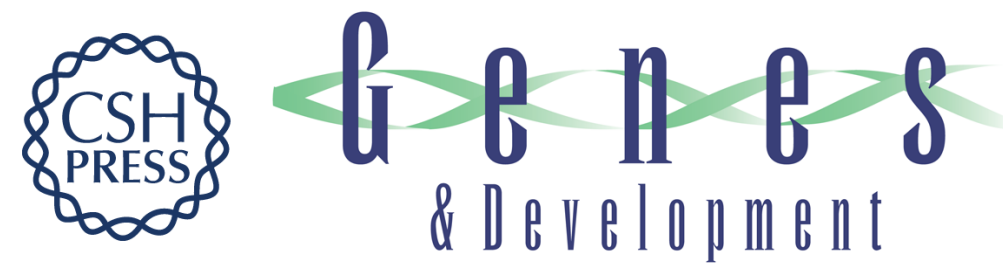

\section{Zebrafish Hairy/Enhancer of split protein links FGF signaling to cyclic gene expression in the periodic segmentation of somites}

Akinori Kawamura, Sumito Koshida, Hiroko Hijikata, et al.

Genes Dev. 2005, 19:

Access the most recent version at doi:10.1101/gad.1291205

Supplemental
Material http://genesdev.cshlp.org/content/suppl/2005/10/20/19.10.1156.DC1

References This article cites 31 articles, 17 of which can be accessed free at:

http://genesdev.cshlp.org/content/19/10/1156.full.html\#ref-list-1

License

Email Alerting

Receive free email alerts when new articles cite this article - sign up in the box at the top

Service

right corner of the article or click here.

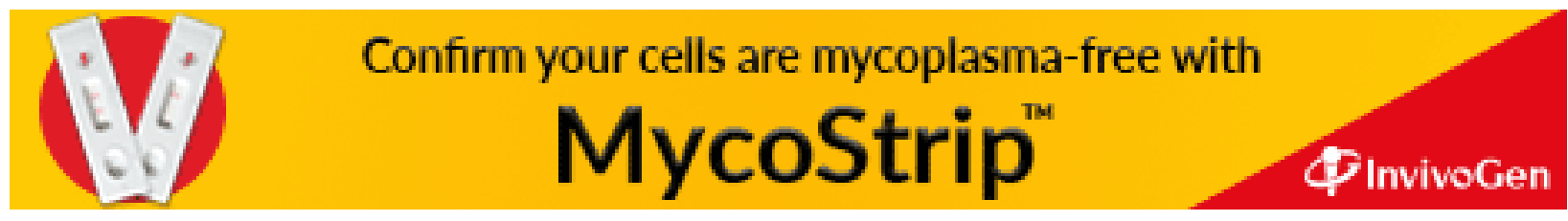

